\title{
The static response of sandwich exact conical helices via MFEM
}

\author{
U.N. Aribas*1 iD, M.H. Omurtag 2 iD \\ ${ }^{1}$ Istanbul Okan University, Department of Civil Engineering, Istanbul, Turkey \\ ${ }^{2}$ Istanbul Medipol University, Department of Civil Engineering, Istanbul, Turkey
}

\begin{abstract}
This paper investigates the internal forces of sandwich exact conical helices using the mixed finite elements based on Timoshenko beam theory and a numerical method of cross-sectional warping inclusion. The exact conical axis geometry is derived over Archimedean and logarithmic planar curves. The exact curved mixed finite element has two nodes and twenty-four degrees of freedom in total. The constitutive equations of sandwich sections are derived by modifying the constitutive equations of an orthotropic continuum based on the classical rod theory. The cross-sectional warping included torsional rigidity is taken into account using a numerical method in the mixed finite element formulation. A convergence analysis for the support reactions of sandwich exact conical helices having fixed-fixed boundary conditions is performed. The results are compared with the displacement type finite element results of computer aided design programs. Finally, the internal forces of sandwich exact conical helices over Archimedean and logarithmic planar curves are obtained for various conicity ratios, pitch angles and the number of active turns. In order to investigate the influence of cross-sectional warping on precision, the warping included results are compared to the conventional torsional rigidity results. In the scope of the investigated internal forces, the maximum influence of cross-sectional warping on the results is obtained for the normal force. The influence of cross-sectional warping on the normal force increases as the pitch angle or the conicity ratio decreases. Besides, the maximum influence of the conical helix geometry on the warping included internal forces is obtained for the torque.
\end{abstract}

\section{Keywords}

Static analysis; Cross-sectional warping; Exact helix geometry; Mixed finite element

Received: 10 October 2019; Accepted: 16 December 2019

ISSN: 2630-5763 (online) C 2019 Golden Light Publishing ${ }^{\circledR}$ All rights reserved.

\section{Introduction}

The composite structural elements are being used extensively in numerous application fields thanks to the recent technology. Since the applied forces mostly cause torsion on the curved structural elements, the inclusion of cross-sectional warping into mathematical operations provides very accurate results. Hans [1] investigated the analogies for the Saint Venant torsion of prismatic solid bars and bending of thin plates. Barretta [2,3] investigated the warping of orthotropic beams by means of the bending twisting of Kirchhoff plates. Barretta et al. [4] investigated the warping of functionally graded beams using the Saint Venant beam theory. Rongqiao et al. [5] expressed the warping of straight bars having orthotropic inhomogeneous rectangular sections analytically. Katsikadelis and Sapountzakis [6] presented a boundary element method for the solution of

\footnotetext{
Corresponding author

E-mail: umit.aribas@okan.edu.tr
} 
torsion for arbitrary shaped composite sections. Li et al. [7] studied the elasticity and finite element solutions for the torsional rigidity of reinforced concrete sections. Sapountzakis [8] investigated the arbitrary composite bars subjected to non-uniform torsion using a boundary element method, and Sapountzakis and Mokos [9] determined the Saint Venant torsional shear and warping included normal stresses. Darılmaz et al. [10] investigated the torsional rigidity of arbitrary composites using an analogy between the deformations of a plate and torsion. Jog and Mokashi [11] investigated the torsion of anisotropic and compound bars using a finite element method based on warping function. Swanson [12] developed a solution for the torsion of laminated cross sections based on the membrane analogy. Fatmi and Zenzri [13] proposed a finite element method for the three-dimensional Saint Venant torsion of isotropic/transversely isotropic composite beams. Fatmi and Ghazouani [14] proposed a higher order composite beam theory to determine the three-dimensional stresses in a larger range of the axial length of the beam with respect to the Saint Venant solution. Barretta [15] studied the relation of twist and shear center of an arbitrary orthotropic Saint Venant beam. Darılmaz et al. [16] proposed a hybrid finite element approach to determine the torsional rigidity and shear stresses of arbitrary composite/functionally graded sections.

The static behavior of composite straight/curved rods are investigated extensively in the literature [17-22]. However, the studies of composite spatial curved rods are quite rare; Y1ldırım [23] investigated the vibrations of composite space Timoshenko rods subjected to thermal conditions. Yıldırım and Sancaktar [24] investigated the free vibrations of symmetrically laminated cross ply composite cylindrical helices based on Timoshenko beam theory. Yildırım $[25,26]$ investigated the free vibrations of composite hyperboloidal, barrel and conical helices using the transfer matrix method based on Timoshenko beam theory. Temel et al. [27] investigated the forced vibration of composite cylindrical helices using the Laplace transformation and complementary functions method based on Timoshenko beam theory. Çalım [28] investigated the free and forced vibrations of composite hyperboloidal and barrel helices based on Timoshenko beam theory. Yu and Hao [29] investigated the free vibrations of symmetrically laminated cross ply composite non cylindrical helices. Yousefi and Rastgoo [30] investigated the free vibrations of functionally graded spatial curved rods based on Timoshenko beam theory. Aribas et al. [31] investigated the warping included free vibration and maximum displacements of composite exact conical helices over either Archimedean and logarithmic planar curve. Besides, the influence of warping on the maximum displacements is investigated.

In this paper, the internal forces of sandwich exact conical helices over Archimedean and logarithmic planar curves are investigated. The first objective of this paper is to compare the crosssectional warping included internal forces to the results obtained using the conventional torsional rigidity. Another objective is to compare the crosssectional warping included internal forces of exact conical helix over Archimedean planar curve to logarithmic planar curve. The exact conical helix geometries are derived using the procedure given in [32]. The analysis is performed using the mixed finite elements based on cross sectional warping included numerical method and Timoshenko beam theory. The two noded exact curved mixed finite elements have twelve degrees of freedom at per node, namely, three displacements, three rotations, three forces and three moments. The constitutive relations of orthotropic composites are derived by modifying constitutive relations of an orthotropic body based on classical rod theory. The inclusion of cross-sectional warping is performed using the numerical method given in [31].

\section{Formulation}

\subsection{The constitutive relations of orthotropic composites}

Letting $\mathbf{E}$ is the elasticity matrix and $\boldsymbol{\sigma}$ and $\varepsilon$ are the stress and strain vectors, respectively, the 
generalized Hooke's law for an orthotropic material is given as $\boldsymbol{\sigma}=\mathbf{E} \boldsymbol{\varepsilon}$. The transformation of the principle material axis around $b$-axis is introduced as $\overline{\boldsymbol{\sigma}}=\left(\mathbf{T}^{-1} \mathbf{E} \mathbf{T}^{-\mathrm{T}}\right) \bar{\varepsilon}=\overline{\mathbf{E}} \bar{\varepsilon} \quad$ [33], where $\mathbf{T}$ is the transformation matrix and $\overline{\mathbf{E}}, \overline{\boldsymbol{\sigma}}$ and $\overline{\boldsymbol{\varepsilon}}$ are the transformed elasticity matrix, stress and strain vectors, respectively. The modification of the constitutive relations for orthotropic body based on the classical rod theory $\sigma_{n}=\sigma_{b}=\tau_{n b}=0$ yields the constitutive relations for a single orthotropic layer [23], where $t, n$ and $b$ are the Frenet coordinates. The constitutive relations of a layer is $\overline{\boldsymbol{\sigma}}_{L}=\overline{\boldsymbol{\beta}}_{L} \overline{\boldsymbol{\varepsilon}}_{L}$ where, $\overline{\boldsymbol{\sigma}}_{L}=\left\{\begin{array}{lll}\sigma_{t} & \tau_{b t} & \tau_{t n}\end{array}\right\}_{L}^{T}$ is the stress vector, $\overline{\boldsymbol{\varepsilon}}_{L}=\left\{\begin{array}{lll}\varepsilon_{t} & \gamma_{b t} & \gamma_{t h}\end{array}\right\}_{L}^{T}$ is the strain vector, $\overline{\boldsymbol{\beta}}_{L}$ is a matrix and $L$ is the number of layers. The displacements at the beam continuum are $u_{t}^{*}=u_{t}+b \Omega_{n}-n \Omega_{b}, \quad u_{n}^{*}=u_{n}-b \Omega_{t} \quad$ and $u_{b}^{*}=u_{b}+n \Omega_{t}$ [30], where $u_{t}, u_{n}$ and $u_{b}$ are displacements on the axis and $\Omega_{t}, \Omega_{n}$ and $\Omega_{b}$ are the rotations. The constitutive relations of a single layer over kinematic relations yield [34],

$$
\left\{\begin{array}{l}
\sigma_{t} \\
\tau_{b t} \\
\tau_{t n}
\end{array}\right\}_{L}=\overline{\boldsymbol{\beta}}_{L}\left\{\left\{\begin{array}{l}
u_{t, t} \\
u_{b, t} \\
u_{n, t}
\end{array}\right\}+b\left\{\begin{array}{c}
\Omega_{n, t} \\
0 \\
-\Omega_{t, t}
\end{array}\right\}+n\left\{\begin{array}{c}
-\Omega_{b, t} \\
\Omega_{t, t} \\
0
\end{array}\right\}\right\}_{L}
$$

where, $L=1, \ldots, N$ is the number of the layer. The commas in the subscripts denote the partial derivations. The analytical integration of stresses through the thickness of cross-section indicates the forces $\left(T_{t}, T_{n}, T_{b}\right)$ and moments $\left(M_{t}, M_{n}, M_{b}\right)$,

$$
\begin{aligned}
T_{t} & =\sum_{L=1}^{N}\left(\int_{-0.5 n_{L}}^{0.5 n_{L}}\left(\int_{b_{L-1}}^{b_{L}} \sigma_{t} \mathrm{~d} b\right) \mathrm{d} n\right) \\
M_{t}= & \sum_{L=1}^{N}\left(\left(-\int_{-0.5 n_{L}}^{0.5 n_{L}}\left(\int_{b_{L-1}}^{b_{L}} b \tau_{t n} \mathrm{~d} b\right) \mathrm{d} n\right)\right. \\
& \left.+\left(\int_{b_{L-1}}^{b_{L}}\left(\int_{-0.5 n_{L}}^{0.5 n_{L}} n \tau_{t b} \mathrm{~d} n\right) \mathrm{d} b\right)\right) \\
T_{n}= & \sum_{L=1}^{N}\left(\int_{-0.5 n_{L}}^{0.5 n_{L}}\left(\int_{b_{L-1}}^{b_{L}} \tau_{t n} \mathrm{~d} b\right) \mathrm{d} n\right)
\end{aligned}
$$

$$
\begin{aligned}
M_{n} & =\sum_{L=1}^{N}\left(\int_{-0.5 n_{L}}^{0.5 n_{L}}\left(\int_{b_{L-1}}^{b_{L}} b \sigma_{t} \mathrm{~d} b\right) \mathrm{d} n\right) \\
T_{b} & =\sum_{L=1}^{N}\left(\int_{-0.5 n_{L}}^{0.5 n_{L}}\left(\int_{b_{L-1}}^{b_{L}} \tau_{b t} \mathrm{~d} b\right) \mathrm{d} n\right) \\
M_{b} & =-\sum_{L=1}^{N}\left(\int_{b_{L-1}}^{b_{L}}\left(\int_{-0.5 n_{L}}^{0.5 n_{L}} n \sigma_{t} \mathrm{~d} n\right) \mathrm{d} b\right)
\end{aligned}
$$

where, the layer's width is $n_{L}$, the coordinates of the $L^{\text {th }}$ layer's bottom and top are $b_{L}$ and $b_{L-1}$ where $b$ indicates positive upward. By means of the Eqn. (2), the constitutive relations of the composite section become,

$$
\left\{\begin{array}{c}
u_{t, t} \\
u_{n, t} \\
u_{b, t} \\
\hdashline \Omega_{t, t} \\
\Omega_{n, t} \\
\Omega_{b, t}
\end{array}\right\}=\left[\begin{array}{c:c}
\mathbf{C}_{m} & \mathbf{C}_{m f} \\
\hdashline \mathbf{C}_{f m} & \mathbf{C}_{f}
\end{array}\right]\left\{\begin{array}{c}
T_{t} \\
T_{n} \\
T_{b} \\
\hdashline M_{t} \\
M_{n} \\
M_{b}
\end{array}\right\}
$$

where, $\mathbf{C}$ is the compliance matrix and $\mathbf{C}_{m f}, \mathbf{C}_{f m}$ are the coupling matrices. The cross-sectional warping included torsional rigidity is determined performing the numerical method given in [31].

\subsection{Functional and mixed finite element formulation}

The exact axial geometry of conical helix is derived using the procedure given in [32]. The field equations of an isotropic curved Timoshenko rod [35] are extended to composites as follows:

$$
\begin{aligned}
& -\mathbf{T}_{, s}-\mathbf{q}=0 \\
& -\mathbf{M}_{, s}-\mathbf{t} \times \mathbf{T}-\mathbf{m}=0 \\
& -\mathbf{C}_{f m} \mathbf{T}-\mathbf{C}_{f} \mathbf{M}+\boldsymbol{\Omega}_{, s}=0 \\
& -\mathbf{C}_{m} \mathbf{T}-\mathbf{C}_{m f} \mathbf{M}+\mathbf{u}_{, s}+\mathbf{t} \times \mathbf{\Omega}=0
\end{aligned}
$$

where the distributed external force and moment vectors are $\mathbf{q}$ and $\mathbf{m}$, respectively. The force and moment vectors are $\mathbf{T}$ and $\mathbf{M}$, respectively. The displacement and cross-sectional rotation vectors are $\mathbf{u}$ and $\boldsymbol{\Omega}$, respectively. The functional is derived via the Gâteaux differential and potential operator concept [36,37], 


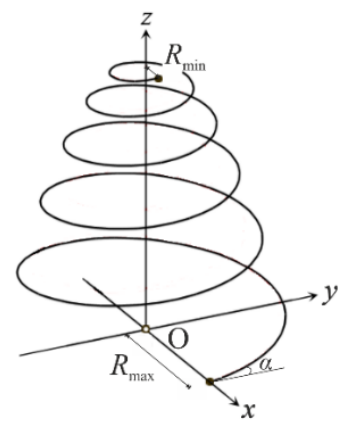

a) Exact conical helix geometry

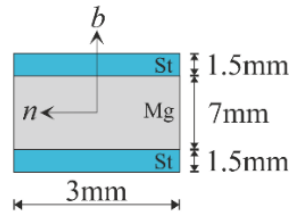

b) Sandwich cross-section

Fig. 1. (a) The exact conical helix geometry, and (b) Sandwich cross-section

$$
\begin{aligned}
I(\mathrm{y})= & -\left[\mathbf{T}_{, s}, \mathbf{u}\right]-\left[\mathbf{M}_{, s}, \mathbf{\Omega}\right]+[\mathbf{t} \times \mathbf{\Omega}, \mathbf{T}] \\
& -\frac{1}{2}\left\{\left[\left(\mathbf{C}_{m}\right) \mathbf{T}, \mathbf{T}\right]+\left[\left(\mathbf{C}_{m f}\right) \mathbf{M}, \mathbf{T}\right]\right. \\
& \left.+\left[\left(\mathbf{C}_{f m}\right) \mathbf{M}, \mathbf{T}\right]+\left[\left(\mathbf{C}_{f}\right) \mathbf{M}, \mathbf{M}\right]\right\} \\
& +[\mathbf{T}, \hat{\mathbf{u}}]_{\varepsilon}+[\mathbf{M}, \hat{\mathbf{\Omega}}]_{\varepsilon}+[(\mathbf{T}-\hat{\mathbf{T}}), \mathbf{u}]_{\sigma} \\
& +[(\mathbf{M}-\hat{\mathbf{M}}), \mathbf{\Omega}]_{\sigma}-[\mathbf{q}, \mathbf{u}]-[\mathbf{m}, \boldsymbol{\Omega}]
\end{aligned}
$$

The linear interpolation functions are used. The two-noded curved mixed finite elements have 24 degrees of freedom in total. The exact functions of curvature, the torsion and the arc length of helix geometry are taken into account. The shear correction factor for Timoshenko beam theory is $5 / 6$.

\section{Numerical examples}

The first objective of numerical examples is to investigate the influence of cross-sectional warping on the internal forces of sandwich exact conical helices over Archimedean and logarithmic planar curves. Another objective is to compare the internal forces of above-mentioned sandwich exact conical helices. The axial geometry is derived using the procedure of general helix given in [32]. The exact conical helix has fixed-fixed boundary conditions. The maximum radius is $R_{\max }=100 \mathrm{~mm}$ and the conicity ratios are $\xi=R_{\min } / R_{\max }=0.8,0.6$ and 0.4

(Fig. 1). Number of active turns is set to $n=2,4$ and 6 for each conicity ratios. The exact conical

helix geometry is derived using constant pitch angles $\alpha=5^{\circ}$ and $20^{\circ}$. The width of the sandwich cross-section is $b=3 \mathrm{~mm}$, the thicknesses of the sandwich cross-section and each face are $h=10$ $\mathrm{mm}$ and $h_{\text {face }}=15 \mathrm{~mm}$, respectively. The faces are made of steel and the core is magnesium. Young's modulus, shear modulus and Poisson's ratio of magnesium are $45 \mathrm{GPa}, 17.442 \mathrm{GPa}$ and 0.29, respectively. The Young's modulus, shear modulus and Poisson ratio of steel are $210 \mathrm{GPa}, 80.769 \mathrm{GPa}$ and 0.3 , respectively.

The exact conical helix is subjected to a uniformly distributed vertical load $0.25 \mathrm{~N} / \mathrm{m}$. 600 mixed finite elements are used in the analysis as it is stated that it provides an excellent agreement with displacement type elements of SAP2000 [31]. The warping included forces $T_{t}, T_{n}, T_{b}$ and moments $M_{t}, M_{n}, M_{b}$ whose absolutes are maximum along the length are given in Tables 1 and 2 for the exact conical helix over Archimedean and logarithmic planar curves, respectively. First, the influence of cross-sectional warping on internal forces is investigated. Then, the warping included internal forces of exact conical helix over Archimedean and logarithmic planar curves are compared to each other.

\subsection{The influence of cross-sectional warping}

\subsubsection{The conicity ratio and pitch angle}

The ratio of warping included internal forces to the internal forces based on Timoshenko beam theory is denoted by $\beta_{w}$ for both exact conical helices. The ratio $\beta_{w}$ for the internal forces $T_{t}, T_{b}, M_{t}$ and $M_{n}$ of exact conical helices is given in Figs. 2 and 3 for the constant pitch angles $\alpha=5^{\circ}$ and $20^{\circ}$, respectively. In the case of $\alpha=5^{\circ}$, The ratio $\beta_{w}>1$ for the internal force $T_{t}$ and it decreases as the conicity ratio $\xi$ increases (Fig. 2a). However, as the constant pitch angle increases $\alpha=20^{\circ}$, the ratio $\beta_{w}<1$ for the increasing number of active turns and it increases as $\xi$ increases (Fig. 3a). 
Table 1. The warping included internal forces $T_{t}, T_{n}, T_{b}$ and moments $M_{t}, M_{n}, M_{b}$ whose absolutes are maximum for exact conical helix over Archimedean planar curve. The forces are $\mathrm{N}$ and moments are $\mathrm{Nm}$.

\begin{tabular}{|c|c|c|c|c|c|c|c|c|}
\hline$\alpha$ & $n$ & $\xi$ & $T_{t}$ & $T_{n}$ & $T_{b}$ & $M_{t}$ & $M_{n}$ & $M_{b}$ \\
\hline \multirow{9}{*}{$5^{\circ}$} & \multirow{3}{*}{2} & 0.4 & 0.0205 & -0.0100 & 0.1285 & -0.0079 & 0.0054 & 0.0010 \\
\hline & & 0.6 & 0.0195 & 0.0084 & 0.1394 & -0.0101 & 0.0060 & -0.0012 \\
\hline & & 0.8 & 0.0183 & -0.0072 & 0.1486 & -0.0127 & 0.0067 & -0.0013 \\
\hline & \multirow{3}{*}{4} & 0.4 & 0.0382 & -0.0153 & 0.2671 & -0.0163 & 0.0063 & 0.0022 \\
\hline & & 0.6 & 0.0359 & -0.0115 & 0.2865 & -0.0208 & 0.0068 & 0.0022 \\
\hline & & 0.8 & 0.0326 & 0.0073 & 0.3012 & -0.0257 & 0.0071 & -0.0025 \\
\hline & \multirow{3}{*}{6} & 0.4 & 0.0520 & 0.0168 & 0.4045 & -0.0247 & 0.0071 & 0.0030 \\
\hline & & 0.6 & 0.0496 & -0.0121 & 0.4325 & -0.0314 & 0.0074 & 0.0032 \\
\hline & & 0.8 & 0.0458 & -0.0069 & 0.4533 & -0.0388 & 0.0075 & -0.0036 \\
\hline \multirow{9}{*}{$20^{\circ}$} & \multirow{3}{*}{2} & 0.4 & 0.0588 & 0.0170 & 0.1205 & -0.0074 & 0.0075 & -0.0024 \\
\hline & & 0.6 & 0.0592 & 0.0138 & 0.1337 & -0.0093 & 0.0078 & -0.0032 \\
\hline & & 0.8 & 0.0585 & 0.0114 & 0.1456 & -0.0113 & 0.0078 & -0.0041 \\
\hline & \multirow{3}{*}{4} & 0.4 & 0.1070 & 0.0118 & 0.2621 & -0.0162 & 0.0091 & 0.0046 \\
\hline & & 0.6 & 0.1110 & -0.0084 & 0.2831 & -0.0204 & 0.0089 & 0.0066 \\
\hline & & 0.8 & 0.1131 & -0.0053 & 0.2995 & -0.0251 & 0.0085 & 0.0087 \\
\hline & \multirow{3}{*}{6} & 0.4 & 0.1547 & 0.0086 & 0.4019 & -0.0247 & 0.0095 & 0.0080 \\
\hline & & 0.6 & 0.1625 & -0.0059 & 0.4309 & -0.0313 & 0.0093 & 0.0107 \\
\hline & & 0.8 & 0.1675 & 0.0033 & 0.4525 & -0.0385 & 0.0087 & 0.0137 \\
\hline
\end{tabular}

Table 2. The warping included internal forces $T_{t}, T_{n}, T_{b}$ and moments $M_{t}, M_{n}, M_{b}$ whose absolutes are maximum for exact conical helix over logarithmic planar curve. The forces are $\mathrm{N}$ and moments are $\mathrm{Nm}$.

\begin{tabular}{|c|c|c|c|c|c|c|c|c|}
\hline$\alpha$ & $n$ & $\xi$ & $T_{t}$ & $T_{n}$ & $T_{b}$ & $M_{t}$ & $M_{n}$ & $M_{b}$ \\
\hline \multirow{9}{*}{$5^{\circ}$} & \multirow{3}{*}{2} & 0.4 & 0.0195 & 0.0097 & 0.1215 & -0.0069 & 0.0053 & 0.0009 \\
\hline & & 0.6 & 0.0193 & -0.0085 & 0.1369 & -0.0098 & 0.0060 & -0.0011 \\
\hline & & 0.8 & 0.0183 & -0.0072 & 0.1480 & -0.0126 & 0.0067 & -0.0013 \\
\hline & \multirow{3}{*}{4} & 0.4 & 0.0369 & -0.0151 & 0.2545 & -0.0146 & 0.0062 & 0.0020 \\
\hline & & 0.6 & 0.0355 & 0.0115 & 0.2817 & -0.0201 & 0.0068 & 0.0021 \\
\hline & & 0.8 & 0.0325 & 0.0074 & 0.3001 & -0.0256 & 0.0071 & -0.0025 \\
\hline & \multirow{3}{*}{6} & 0.4 & 0.0502 & -0.0167 & 0.3861 & -0.0222 & 0.0070 & 0.0028 \\
\hline & & 0.6 & 0.0490 & -0.0121 & 0.4255 & -0.0305 & 0.0074 & 0.0031 \\
\hline & & 0.8 & 0.0457 & -0.0070 & 0.4516 & -0.0386 & 0.0075 & -0.0036 \\
\hline \multirow{9}{*}{$20^{\circ}$} & \multirow{3}{*}{2} & 0.4 & 0.0556 & 0.0166 & 0.1138 & -0.0063 & 0.0070 & -0.0021 \\
\hline & & 0.6 & 0.0582 & 0.0139 & 0.1312 & -0.0089 & 0.0077 & -0.0031 \\
\hline & & 0.8 & 0.0584 & -0.0115 & 0.1451 & -0.0112 & 0.0078 & -0.0041 \\
\hline & \multirow{3}{*}{4} & 0.4 & 0.1021 & -0.0118 & 0.2492 & -0.0143 & 0.0088 & -0.0042 \\
\hline & & 0.6 & 0.1092 & -0.0084 & 0.2783 & -0.0197 & 0.0089 & 0.0063 \\
\hline & & 0.8 & 0.1127 & 0.0053 & 0.2984 & -0.0249 & 0.0085 & 0.0086 \\
\hline & \multirow{3}{*}{6} & 0.4 & 0.1479 & -0.0086 & 0.3833 & -0.0221 & 0.0093 & 0.0070 \\
\hline & & 0.6 & 0.1599 & -0.0060 & 0.4238 & -0.0303 & 0.0093 & 0.0103 \\
\hline & & 0.8 & 0.1669 & 0.0033 & 0.4508 & -0.0383 & 0.0087 & 0.0136 \\
\hline
\end{tabular}




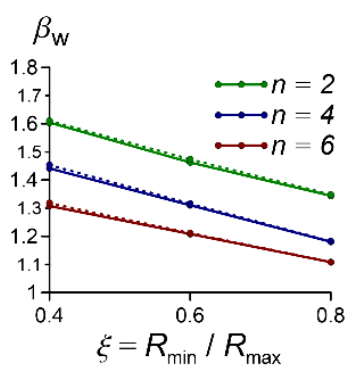

a) $T_{t}$

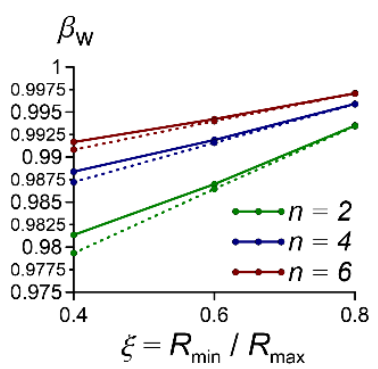

b) $T_{b}$

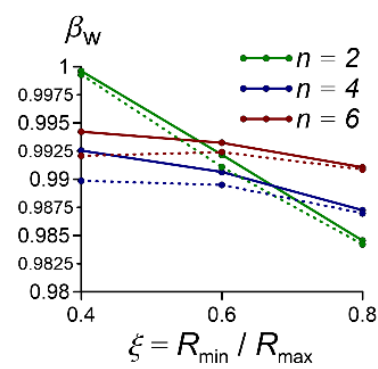

c) $M_{t}$

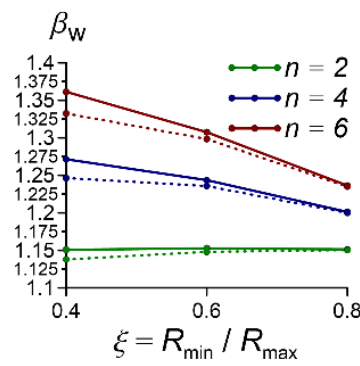

d) $M_{n}$

Fig. 2. The ratio $\beta_{w}$ of internal forces for the conicity ratio $\xi$. Continuous and dashed lines are used for Archimedean and logarithmic planar curves, respectively. $\left(\alpha=5^{\circ}\right)$

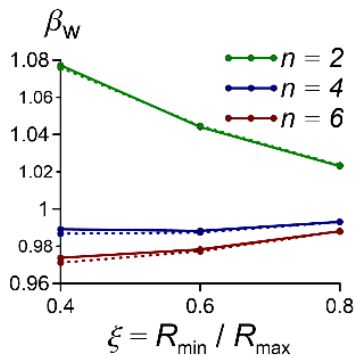

a) $T_{t}$

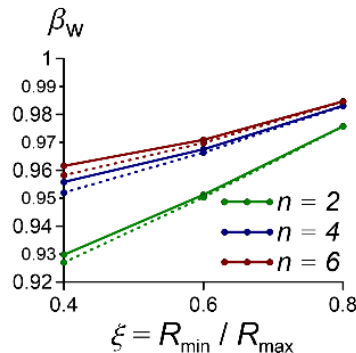

b) $T_{b}$

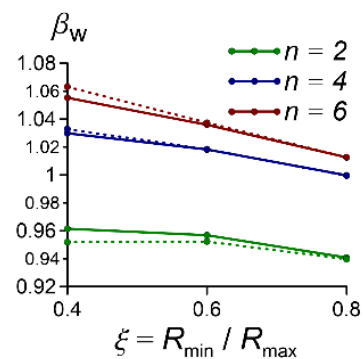

c) $M_{t}$

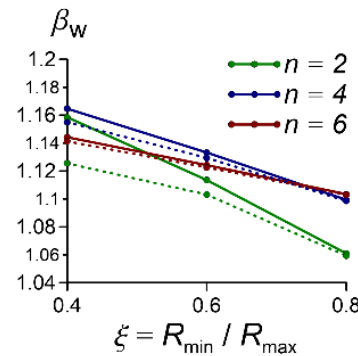

d) $M_{n}$

Fig. 3. The ratio $\beta_{w}$ of internal forces for the conicity ratio $\xi$. Continuous and dashed lines are used for Archimedean and logarithmic planar curves, respectively. $\left(\alpha=20^{\circ}\right)$

In the case of $T_{b}$, the ratio $\beta_{w}<1$ both for the constant pitch angles $\alpha=5^{\circ}$ and $20^{\circ}$, and it decreases more as $\alpha$ increases (Figs. 2b and 3b). The ratio $\beta_{w}$ for the internal force $T_{b}$ increases as $\xi$ increases. In the case of $\alpha=5^{\circ}$, the ratio $\beta_{w}<1$ for the internal force $M_{t}$ and it decreases as $\xi$ increases (Fig. 2c). However, as the constant pitch angle increases $\alpha=20^{\circ}$, the ratio $\beta_{w}>1$ for the increasing number of active turns (Fig. 3c). In the case of both constant pitch angles $\alpha=5^{\circ}$ and $20^{\circ}$, the ratio $\beta_{w}>1$ for the internal force $M_{n}$ and it has an decreasing tendency as the number of active turns $n$ increases. Within the scope of given internal forces, the maximum ratio $\beta_{w}=1.611$ is obtained for the internal force $T_{t}$.

\subsubsection{The number of active turns and pitch angle}

In the case of increasing number of active turns $n$, the ratio $\beta_{w}$ for the internal forces $T_{t}, T_{b}, M_{t}$ and $M_{n}$ of exact conical helices is given in
Figures 4 and 5 for the constant pitch angles $\alpha=5^{\circ}$ and $20^{\circ}$, respectively. In the case of $\alpha=5^{\circ}$, the ratio $\beta_{w}>1$ for the internal force $T_{t}$ and it decreases as $n$ increases (Fig. 4a). Besides, as the constant pitch angle increases $\alpha=20^{\circ}$, the ratio $\beta_{w}$ for the internal force $T_{t}$ decreases more (Fig. 5a). In the case of internal force $T_{b}$, the ratio $\beta_{w}<1$ both for the constant pitch angles $\alpha=5^{\circ}$ and $20^{\circ}$, and it decreases more as $\alpha$ increases (Figs. $4 \mathrm{~b}$ and 5b). The ratio $\beta_{w}$ for the internal force $T_{b}$ increases as the number of active turns $n$ increases. The ratio $\beta_{w}$ for the internal force $M_{t}$ has an increasing tendency as $n$ increases (Figs. 4c and 5c). In the case of the internal force $M_{n}$, the ratio $\beta_{w}>1$ both for the constant pitch angles $\alpha=5^{\circ}$ and $20^{\circ}$. In the case of $\alpha=5^{\circ}$, the ratio $\beta_{w}$ for the internal force $M_{n}$ increases as $n$ increases (Fig. 4d). 


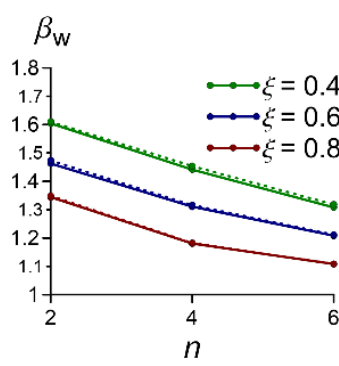

a) $T_{t}$

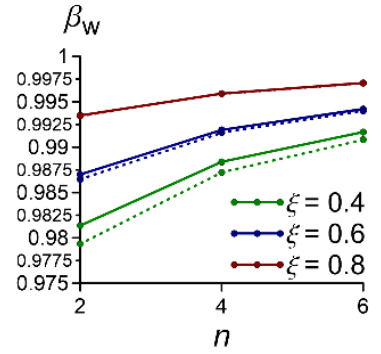

b) $T_{b}$

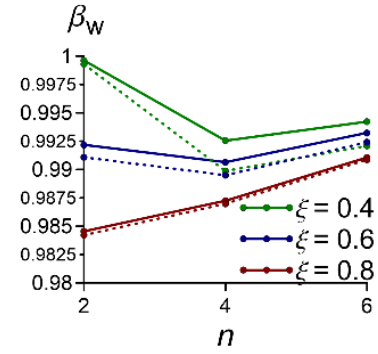

c) $M_{t}$

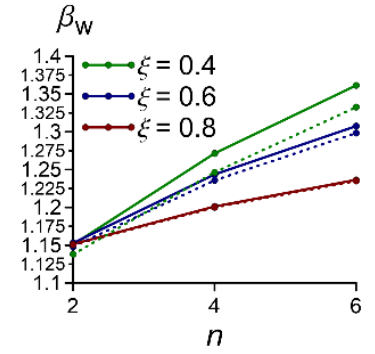

d) $M_{n}$

Fig. 4. The ratio $\beta_{w}$ of internal forces for the number of active turns $n$. Continuous and dashed lines are used for Archimedean and logarithmic planar curves, respectively. $\left(\alpha=5^{\circ}\right)$

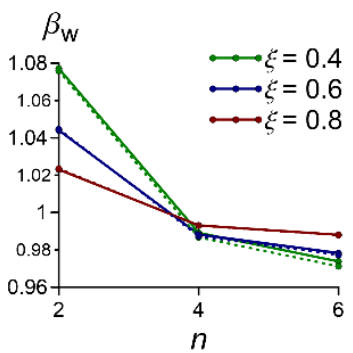

a) $T_{t}$

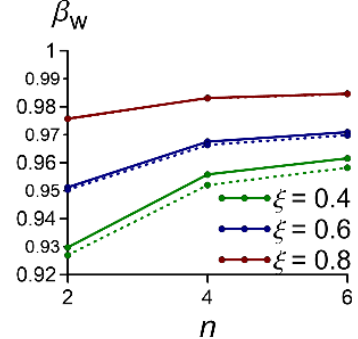

b) $T_{b}$

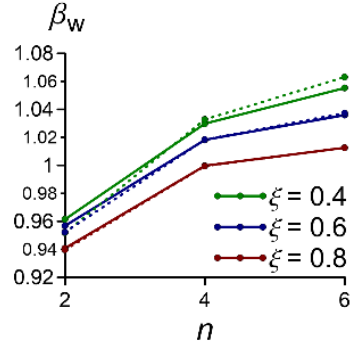

c) $M_{t}$

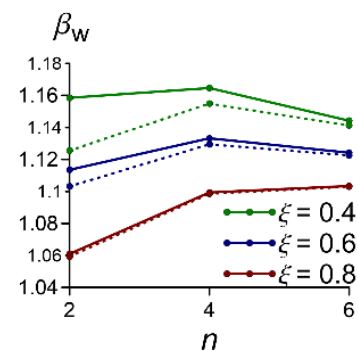

d) $M_{n}$

Fig. 5. The ratio $\beta_{w}$ of internal forces for the number of active turns $n$. Continuous and dashed lines are used for

Archimedean and logarithmic planar curves, respectively. $\left(\alpha=20^{\circ}\right)$

However, in the case of $\alpha=20^{\circ}$, it has a decreasing tendency as $n$ increases and $\xi$ decreases (Fig. 5d).

\subsection{The exact conical helix geometry}

\subsubsection{The conicity ratio and pitch angle}

The ratio of cross-sectional warping included internal forces of exact conical helix over Archimedean planar curve to logarithmic planar curve is denoted by $\beta_{g}$. The ratio $\beta_{g}$ for the internal forces $T_{t}, T_{b}, M_{t}$ and $M_{n}$ of exact conical helices is given in Figs. 6 and 7 for the constant pitch angles $\alpha=5^{\circ}$ and $20^{\circ}$, respectively. In the cases of the internal forces $T_{t}, T_{b}$ and $M_{t}$ , the ratio $\beta_{g}>1$ both for the constant pitch angles $\alpha=5^{\circ}$ and $20^{\circ}$, and it decreases as the conicity ratio $\xi$ increases (Figs. 2a-c and 3a-c). The ratio $\beta_{g}$ for the internal force $M_{n}$ has an increasing tendency as $\alpha$ decreases and the number of active turns $\xi$ increases (Figs. 6d and 7d). Within the scope of the given internal forces, the maximum ratio $\beta_{g}=1.172$ is obtained for the internal force $M_{t}$.

\subsubsection{The number of active turns and pitch angle}

In the case of increasing number of active turns $n$, the ratio $\beta_{g}$ for the internal forces $T_{t}, T_{b}, M_{t}$ and $M_{n}$ of exact conical helices is given in Figs. 8 and 9 for the constant pitch angles $\alpha=5^{\circ}$ and $20^{\circ}$, respectively. The ratio $\beta_{g}>1$ for the internal forces $T_{t}, T_{b}$ and $M_{t}$ (Figs. 8a-c and 9a-c). However, in the case of decreasing conicity ratio $(\xi=0.4)$, it has a decreasing tendency as the number of active turns $n$ increases. In the case of increasing conicity ratio $(\xi=0.8)$, the ratio $\beta_{g}$ for the internal force $M_{n}$ has an increasing tendency as $n$ increases (Figs. 8d and 9d). As the constant pitch angle $\alpha$ increases, the ratio $\beta_{g}$ for the internal force $M_{n}$ increases. 


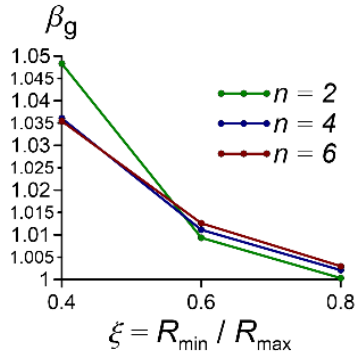

a) $T_{t}$

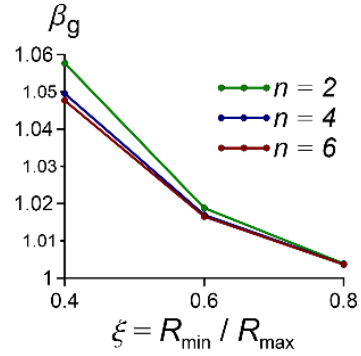

b) $T_{b}$

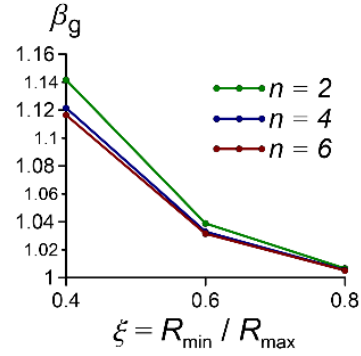

c) $M_{t}$

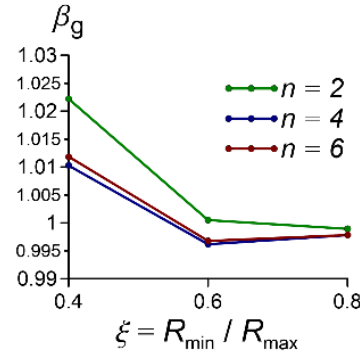

d) $M_{n}$

Fig. 6. The ratio $\beta_{g}$ of internal forces for the conicity ratio $\xi,\left(\alpha=5^{\circ}\right)$.

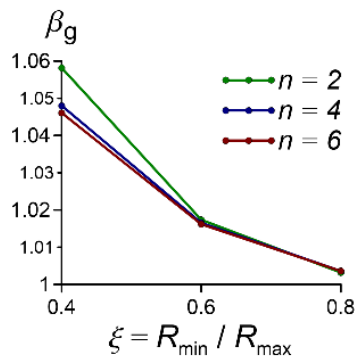

a) $T_{t}$

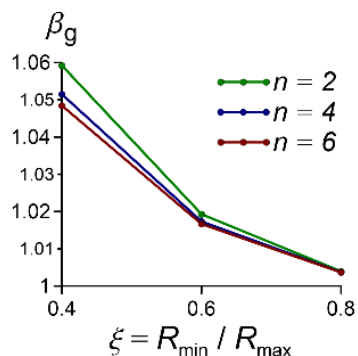

b) $T_{b}$

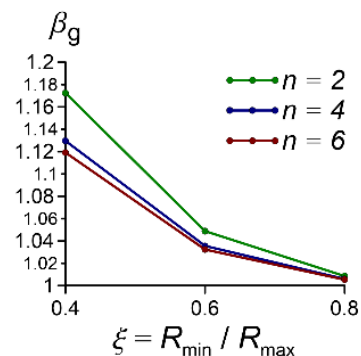

c) $M_{t}$

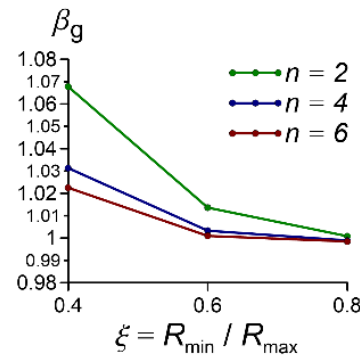

d) $M_{n}$

Fig. 7. The ratio $\beta_{g}$ of internal forces for the conicity ratio $\xi,\left(\alpha=20^{\circ}\right)$.

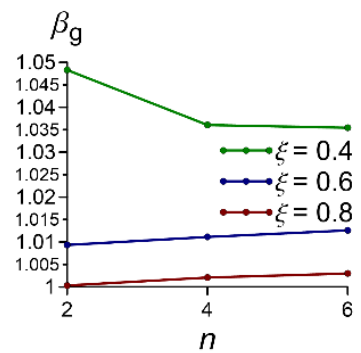

a) $T_{t}$

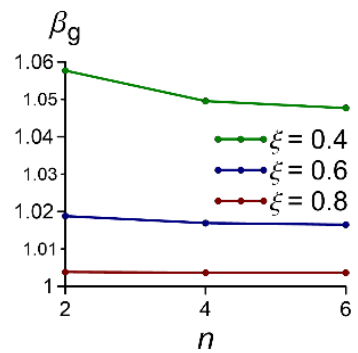

b) $T_{b}$

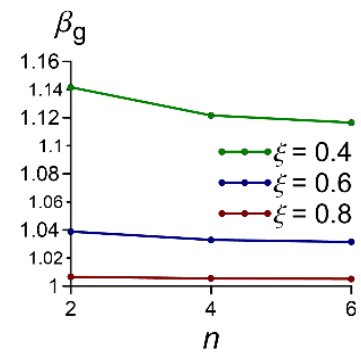

c) $M_{t}$

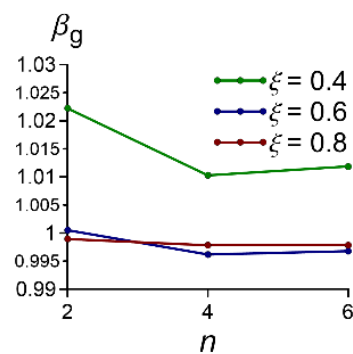

d) $M_{n}$

Fig. 8. The ratio $\beta_{g}$ of internal forces for the number of active turns $n,\left(\alpha=5^{\circ}\right)$.

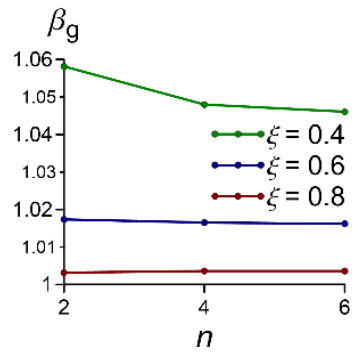

a) $T_{t}$

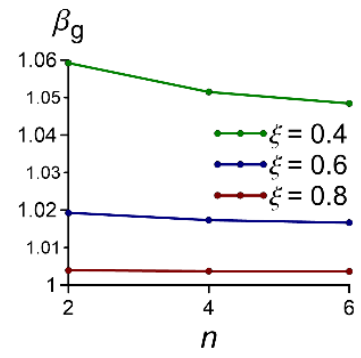

b) $T_{b}$

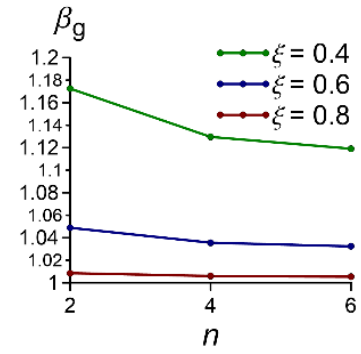

c) $M_{t}$

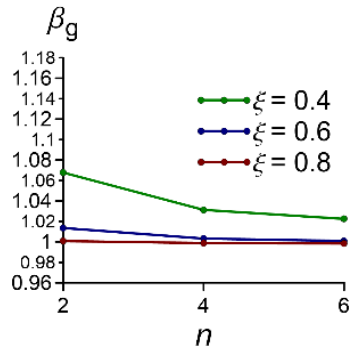

d) $M_{n}$

Fig. 9. The ratio $\beta_{g}$ of internal forces for the number of active turns $n,\left(\alpha=20^{\circ}\right)$. 


\section{Conclusions}

The influence of cross-sectional warping on the internal forces of exact conical helices is investigated using the mixed finite elements based on a numerical method of warping inclusion and Timoshenko beam theory. The exact axial geometry of conical helix over Archimedean and logarithmic planar curves is derived using the procedure given in [32]. The warping included torsional rigidity of composite non-circular cross-sections is determined using the numerical method given in [31]. The ratio of cross-sectional warping included internal forces of exact conical helices to the internal forces based on Timoshenko beam theory is denoted as $\beta_{w}$. The ratio of warping included internal forces of exact conical helix over Archimedean planar curve to logarithmic planar curve is denoted as $\beta_{g}$.

- The normal force $T_{t}$ is the most influenced internal force from warping effect within the investigated internal forces.

- The difference between the normal forces obtained via Timoshenko beam theory and the numerical method of warping inclusion increases as the conicity ratio and/or the number of active turns decreases. The warping included normal force becomes greater than the normal force obtained via Timoshenko beam theory as the pitch angle decreases.

- In the case of smaller degrees of pitch angle, the difference between the torques obtained via Timoshenko beam theory and the numerical method of warping inclusion is directly proportional with the conicity ratio. However, in the case of greater degrees of pitch angle, it becomes inversely proportional with the conicity ratio, especially as the number of active turns increases.

- The torque $M_{t}$ is the most influenced internal force from the conical helix geometry within the investigated internal forces.

- The difference between the warping included internal forces $T_{t}, T_{b}$ and $M_{t}$ of exact conical helices over Archimedean and logarithmic planar curves increases as the conicity ratio decreases. The increasing tendency is independent from the pitch angle.

As the number of active turns and conicity ratio decrease, the difference between the warping included internal forces of exact conical helices over Archimedean and logarithmic planar curves has an increasing tendency.

\section{Acknowledgment}

The authors gratefully acknowledge Prof. Dr. Nihal Eratl1, Asst. Prof. Dr. Akif Kutlu and Dr. Merve Ermiş.

\section{References}

[1] Irschik Hans (1991) Analogies between Bending of Plates and Torsion Problem. Journal of Engineering Mechanics 117: 2503-2508.

[2] Barretta R (2014) Analogies between Kirchhoff plates and Saint-Venant beams under flexure. Acta Mechanica; Wien 225: 2075-2083.

[3] Barretta R (2013) Analogies between Kirchhoff plates and Saint-Venant beams under torsion. Acta Mech. 224: 2955-2964.

[4] Barretta R, Feo L, Luciano R (2015) Some closedform solutions of functionally graded beams undergoing nonuniform torsion. Composite Structures 123: 132-136.

[5] Rongqiao X, Jiansheng H, Weiqiu C (2010) SaintVenant torsion of orthotropic bars with inhomogeneous rectangular cross section. Composite Structures 92: 1449-1457.

[6] Katsikadelis JT, Sapountzakis EJ (1985) Torsion of composite bars by boundary element method. Journal of Engineering Mechanics 111: 11971210.

[7] Li Z, Ko JM, Ni YQ (2000) Torsional rigidity of reinforced concrete bars with arbitrary sectional shape. Finite Elements in Analysis and Design 35: 349-361.

[8] Sapountzakis EJ (2001) Nonuniform torsion of multi-material composite bars by the boundary element method. Computers \& Structures 79: 2805-2816.

[9] Sapountzakis EJ, Mokos VG (2003) Warping shear stresses in nonuniform torsion of composite bars by BEM. Computer Methods in Applied Mechanics and Engineering 192: 4337-4353. doi:10/dcgs6m. 
[10] Darılmaz K, Orakdöğen E, Girgin K, Kucukarslan S (2007) Torsional rigidity of arbitrarily shaped composite sections by hybrid finite element approach. Steel and Composite Structures 7: 241251.

[11] Jog CS, Mokashi IS (2014) A finite element method for the Saint-Venant torsion and bending problems for prismatic beams. Computers \& Structures 135: 62-72.

[12] Swanson SR (1998) Torsion of laminated rectangular rods. Composite Structures 4223-31.

[13] El Fatmi R, Zenzri H (2004) A numerical method for the exact elastic beam theory. Applications to homogeneous and composite beams. International Journal of Solids and Structures 41: 2521-2537.

[14] El Fatmi R, Ghazouani N (2011) Higher order composite beam theory built on Saint-Venant's solution. Part-I: Theoretical developments. Composite Structures 93557-566.

[15] Barretta R (2012) On the relative position of twist and shear centres in the orthotropic and fiberwise homogeneous Saint-Venant beam theory. International Journal of Solids and Structures 49: 3038-3046.

[16] Darılmaz K, Orakdöğen E, Girgin K (2018) SaintVenant torsion of arbitrarily shaped orthotropic composite or FGM sections by a hybrid finite element approach. Acta Mech. 229: 1387-1398.

[17] Khdeir AA, Reddy JN (1997) An exact solution for the bending of thin and thick cross-ply laminated beams. Composite Structures 37: 195-203.

[18] Kennedy GJ, Hansen JS, Martins JRRA (2011) A Timoshenko beam theory with pressure corrections for layered orthotropic beams. International Journal of Solids and Structures 48: 2373-2382.

[19] Aguiar RM, Moleiro F, Mota Soares CM (2012) Assessment of mixed and displacement-based models for static analysis of composite beams of different cross-sections. Composite Structures 94: 601-616.

[20] Vo TP, Thai HT (2012) Static behavior of composite beams using various refined shear deformation theories. Composite Structures 94: 2513-2522.

[21] Babuska P, Wiebe R, Motley MR (2018) A beam finite element for analysis of composite beams with the inclusion of bend-twist coupling. Composite Structures 189: 707-717.

[22] Nguyen TK, Nguyen ND, Vo TP, Thai HT (2017) Trigonometric-series solution for analysis of laminated composite beams. Composite Structures 160: $142-151$.

[23] Yıldırım V (1999) Governing equations of initially twisted elastic space rods made of laminated composite materials. International Journal of Engineering Science 37: 1007-1035.

[24] Yildırım V, Sancaktar E (2000) Linear free vibration analysis of cross-ply laminated cylindrical helical springs. International Journal of Mechanical Sciences 42: 1153-1169.

[25] Y1ldırım V (2001) Free vibration characteristics of composite barrel and hyperboloidal coil springs. Mechanics of Composite Materials and Structures 8: 205-217.

[26] Y1ldirım V (2004) A parametric study on the natural frequencies of unidirectional composite conical springs. Communications in Numerical Methods in Engineering 20: 207-227.

[27] Temel B, Çalım FF, Tütüncü N (2005) Forced vibration of composite cylindrical helical rods. International Journal of Mechanical Sciences 47: 998-1022.

[28] Çalım FF (2009) Dynamic analysis of composite coil springs of arbitrary shape. Composites Part B: Engineering 40: 741-757.

[29] Yu A, Hao Y (2013) Effect of warping on natural frequencies of symmetrical cross-ply laminated composite non-cylindrical helical springs. International Journal of Mechanical Sciences 74: 65-72.

[30] Yousefi A, Rastgoo A (2011) Free vibration of functionally graded spatial curved beams. Composite Structures 93: 3048-3056.

[31] Aribas UN, Ermis M, Eratli N, Omurtag MH (2019) The static and dynamic analyses of warping included composite exact conical helix by mixed FEM. Composites Part B: Engineering 160: 285297.

[32] Ermis M, Omurtag MH (2017) Static and dynamic analysis of conical helices based on exact geometry via mixed FEM. International Journal of Mechanical Sciences 131-132: 296-304.

[33] Jones RM. Mechanics of composite materials. 2nd ed. Taylor \& Francis, Philadelphia, PA, 1999.

[34] Bhimaraddi A, Chandrashekhara K (1991) Some observations on the modeling of laminated composite beams with general lay-ups. Composite Structures 19: 371-380.

[35] Omurtag MH, Aköz AY (1992) The mixed finite element solution of helical beams with variable 
cross-section under arbitrary loading. Computers \& Structures 43: 325-331.

[36] Oden JT, Reddy JN. Variational methods in theoretical mechanics. Springer Science \& Business Media, 2012.
[37] Omurtag MH, Aköz AY (1994) Hyperbolic paraboloid shell analysis via mixed finite element formulation. International Journal for Numerical Methods in Engineering 37: 3037-3056. 